Delft University of Technology

\title{
Ovarian Cancer-Specific BRCA-like Copy-Number Aberration Classifiers Detect Mutations Associated with Homologous Recombination Deficiency in the AGO-TR1 Trial
}

Schouten, Philip C.; Richters, Lisa; Vis, Daniel J.; Kommoss, Stefan; Kluin, Roelof J.C.; Lips, Esther H.; Schmidt, Sandra; Nederlof, Petra M.; Wessels, Lodewyk F.; More Authors

DOI

10.1158/1078-0432.CCR-21-1673

\section{Publication date}

2021

Document Version

Final published version

Published in

Clinical Cancer Research

\section{Citation (APA)}

Schouten, P. C., Richters, L., Vis, D. J., Kommoss, S., Kluin, R. J. C., Lips, E. H., Schmidt, S., Nederlof, P. M., Wessels, L. F., \& More Authors (2021). Ovarian Cancer-Specific BRCA-like Copy-Number Aberration Classifiers Detect Mutations Associated with Homologous Recombination Deficiency in the AGO-TR1 Trial. Clinical Cancer Research, 27(23), 6559-6569. https://doi.org/10.1158/1078-0432.CCR-21-1673

\section{Important note}

To cite this publication, please use the final published version (if applicable).

Please check the document version above.

\section{Copyright}

Other than for strictly personal use, it is not permitted to download, forward or distribute the text or part of it, without the consent of the author(s) and/or copyright holder(s), unless the work is under an open content license such as Creative Commons. 


\section{Ovarian Cancer-Specific BRCA-like Copy-Number Aberration Classifiers Detect Mutations Associated with Homologous Recombination Deficiency in the AGO-TR1 Trial}

Philip C. Schouten', Lisa Richters², Daniel J. Vis ${ }^{3}$, Stefan Kommoss ${ }^{4}$, Ewald van Dijk ${ }^{3}$, Corinna Ernst ${ }^{2}$, Roelof J.C. Kluin ${ }^{5}$, Frederik Marmé ${ }^{6}$, Esther H. Lips', Sandra Schmidt' ${ }^{1}$, Esther Scheerman, Katharina Prieske ${ }^{8}$, Carolien H.M. van Deurzen ${ }^{9}$, Alexander Burges ${ }^{10}$, Patricia C. Ewing-Graham ${ }^{9}$, Dimo Dietrich" ${ }^{11}$, Agnes Jager ${ }^{12}$, Nikolaus de Gregorio ${ }^{13}$, Jan Hauke ${ }^{2}$, Andreas du Bois ${ }^{14}$, Petra M. Nederlof ${ }^{7}$, Lodewyk F. Wessels ${ }^{3,15}$, Eric Hahnen², Philipp Harter ${ }^{14}$, Sabine C. Linn ${ }^{1,16,17}$, and Rita K. Schmutzler ${ }^{2}$

\section{ABSTRACT}

Purpose: Previously, we developed breast cancer BRCA1-like and BRCA2-like copy-number profile shrunken centroid classifiers predictive for mutation status and response to therapy, targeting homologous recombination deficiency (HRD). Therefore, we investigated BRCA1- and BRCA2-like classification in ovarian cancer, aiming to acquire classifiers with similar properties as those in breast cancer.

Experimental Design: We analyzed DNA copy-number profiles of germline BRCA1- and BRCA2-mutant ovarian cancers and control tumors and observed that existing breast cancer classifiers did not sufficiently predict mutation status. Hence, we trained new shrunken centroid classifiers on this set and validated them in the independent The Cancer Genome Atlas dataset. Subsequently, we assessed BRCA1/2-like classification and obtained germline and tumor mutation and methylation status of cancer predisposition

\section{Introduction}

Epithelial ovarian cancer is the second leading cause of death among gynecologic cancers worldwide as there were 286,000 incident cases and mortality of 176,000 reported in 2017 (1). Because screening tools are ineffective and early clinical warning signs are rare, the majority of ovarian cancer cases present in late clinical

'Department of Molecular Pathology, Netherlands Cancer Institute, Amsterdam, the Netherlands. ${ }^{2}$ Center for Familial Breast and Ovarian Cancer, Center for Integrated Oncology (CIO), Medical Faculty, University Hospital Cologne, Cologne, Germany. ${ }^{3}$ Department of Molecular Carcinogenesis, Netherlands Cancer Institute, Amsterdam, the Netherlands. ${ }^{4}$ Department of Women's Health, Tübingen University Hospital, Tübingen, Germany. ${ }^{5}$ Genomics Core Facility, Netherlands Cancer Institute-Antoni van Leeuwenhoek Hospital, Amsterdam, the Netherlands. ${ }^{6}$ Department of Gynecologic Oncology, Medical Faculty Mannheim, University of Heidelberg, University Hospital Mannheim, Mannheim, Germany. ${ }^{7}$ Department of Pathology, Netherlands Cancer Institute, Amsterdam, the Netherlands. ${ }^{8}$ Department of Gynecology and Gynecologic Oncology, University Medical Center Hamburg-Eppendorf, Hamburg, Germany. ${ }^{9}$ Department of Pathology, Erasmus MC, Rotterdam, the Netherlands. ${ }^{10}$ Department of Gynecology and Obstetrics, University Hospital Munich-Großhadern, Munich, Germany. "Department of Otolaryngology, Head and Neck Surgery, University Hospital Bonn, Bonn, Germany. ${ }^{12}$ Department of Medical Oncology, Erasmus MC, Rotterdam, the Netherlands. ${ }^{13}$ Department of Gynecology and Obstetrics, University Hospital, University of Ulm, Ulm, Germany. ${ }^{14}$ Department of Gynecology and Gynecologic Oncology, Ev. Kliniken Essen-Mitte, Essen, Germany. ${ }^{15}$ Faculty of Electrical Engineering, Mathematics and Com- genes, among them several involved in HR repair, of 300 ovarian cancer samples derived from the consecutive cohort trial AGO-TR1 (NCT02222883).

Results: The detection rate of the BRCA1-like classifier for BRCA1 mutations and promoter hypermethylation was $95.6 \%$. The BRCA2-like classifier performed less accurately, likely due to a smaller training set. Furthermore, three quarters of the BRCA1/ 2-like tumors could be explained by (epi)genetic alterations in $B R C A 1 / 2$, germline $R A D 51 C$ mutations and alterations in other genes involved in HR. Around half of the non-BRCA-mutated ovarian cancer cases displayed a $B R C A$-like phenotype.

Conclusions: The newly trained classifiers detected most $B R C A$ mutated and methylated cancers and all tumors harboring a RAD51C germline mutations. Beyond that, we found an additional substantial proportion of ovarian cancers to be BRCA-like.

stages. Even though therapeutic strategies improved within the past years, the prognosis is still poor, with an average 5-year survival rate of $48.6 \%$ (2).

Cytoreductive surgery aiming for complete resection, followed by platinum-based chemotherapy, has been the backbone of ovarian cancer treatment for decades $(3,4)$. Carboplatin, combined with paclitaxel \pm bevacizumab, as an initial systemic regimen leads to a

puter Science, Delft University of Technology, Delft, the Netherlands. ${ }^{16}$ Department of Medical Oncology, Netherlands Cancer Institute, Amsterdam, the Netherlands. ${ }^{17}$ Department of Pathology, University Medical Center Utrecht, Utrecht, the Netherlands.

Note: Supplementary data for this article are available at Clinical Cancer Research Online (http://clincancerres.aacrjournals.org/).

P.C. Schouten and L. Richters contributed equally to this article as first authors. P. Harter, S.C. Linn, and R.K. Schmutzler contributed equally to this article as last authors.

Corresponding Author: Philip C. Schouten, Department of Molecular Pathology, Netherlands Cancer Institute, Plesmanlaan 121, 1066CX Amsterdam, the Netherlands. Phone: 312-051-2449; E-mail: p.schouten@nki.nl

Clin Cancer Res 2021;27:6559-69

doi: 10.1158/1078-0432.CCR-21-1673

This open access article is distributed under Creative Commons AttributionNonCommercial-NoDerivatives License 4.0 International (CC BY-NC-ND).

(C)2021 The Authors; Published by the American Association for Cancer Research 


\section{Translational Relevance}

We developed ovarian cancer-specific DNA copy-number aberration classifiers that identify a population enriched for BRCA1/2 and HR-associated gene mutations and might be promising biomarker candidates for response to HRD-directed treatment.

response rate of approximately $80 \%$, especially in high-grade serous ovarian cancer (HGSOC). Nevertheless, the disease of most patients recurs over time (5).

Recently, PARP inhibitors (PARPi) were added to the therapeutic arsenal. Both the mainstay carboplatin/paclitaxel and these new strategies yield high responses in the overall population, which could be explained by homologous recombination deficiency (HRD) in a substantial proportion of ovarian cancer (6).

Platinum compounds and PARPi exploit the HRD, by inducing DNA double-strand breaks or impeding its repair via synthetic lethality, leading to cell-cycle arrest or death. The breast and ovarian cancer germline predisposition genes $B R C A 1$ and $B R C A 2$ play crucial roles in homologous recombination $(7,8)$, an essential, highly accurate DNA-repair process fixing double-strand breaks. Deleterious germline mutations with subsequent loss of heterozygosity (LOH) in BRCA1/2 can explain a subset of HR-deficient ovarian cancers, resulting in the registration of $B R C A$ mutation analyses as companion diagnostics in specific ovarian cancer (9) and metastasized breast cancer (10) settings. However, it was found that a larger proportion of ovarian cancer displays a phenotype similar to germline (g) BRCA1/2-mutated cancers, so-called BRCAness (11). This is supported by preclinical analyses (12) and clinical trials of the three registered PARPi in ovarian cancer (13-16) demonstrating survival benefits for a larger subgroup, and has led to their approval, independent of mutation status in most indications. Because these additional patients might benefit from a specific therapy, various potential biomarkers are under investigation, including single gene methylation, gene expression (profiles), copy number/LOH-based assays, mutational signatures, and combinations (17-21).

Of these, the LOH score (ref. 15; Foundation Medicine) and MyChoice (ref. 22; Myriad) were applied within the trials mentioned above and demonstrated the ability to narrow the respective subgroup. This led to the first approval of an HRD assay (MyChoice) as a companion diagnostic for applying niraparib in heavily pretreated patients with ovarian cancer (23). Two recent clinical trials $(24,25)$ evaluated this biomarker in the first-line setting, showing a benefit of a PARPi predominantly in the HRD-positive, but also in the HRDnegative subgroup. In contrast, within the PAOLA-1 trial (26), the test defined a subpopulation beyond $B R C A$ mutation carriers benefitting most from the addition of olaparib to bevacizumab maintenance therapy after carboplatin and paclitaxel. Only recently, the European Medicines Agency recommended the approval of this combination therapy for HR-deficient ovarian cancer defined by the presence of $B R C A$ mutations or genomic instability for first-line maintenance treatment accordingly (27). Nonetheless, the exploration of further transparent tests that can easily be implemented and further elaborated on decentral platforms is still ongoing to improve the quest for predictive markers.

In breast cancer, a $B R C A$-like classifier based on tissue-specific copynumber profiles was built (28) to discriminate BRCA-associated from sporadic cancers by employing the shrunken centroid algorithm (29). This classifier was successfully validated as a predictive marker for benefit of high-dose alkylating chemotherapy in stage III HER2/neunegative breast cancer $(30,31)$, which could be confirmed in several independent retrospective cohorts $(32,33)$ and is in validation within a prospective breast cancer trial (34). Its derivative gene expression classifier predicted response to a combination of neoadjuvant veliparib and carboplatin (35) in triple-negative breast cancer.

We apply the same methodologic approach to create ovarian cancer-specific BRCA1/2-like classifiers, assuming them to be reliable screening tools for HRD in ovarian cancer. In the current study, we further validate these classifiers within The Cancer Genome Atlas (TCGA) cohort and the independent, well-characterized sample set of the observational AGO-TR1 trial (NCT02222883; refs. 36, 37).

The main goal was to evaluate their predictive power in finding samples with a known HR defect (i.e., BRCA-associated tumors). Moreover, we want to identify other genetic and clinical characteristics associated with a BRCA-like profile and investigate this phenotype's occurrence within the examined cohorts.

\section{Materials and Methods}

\section{Ovarian cancer-BRCA1/2-like classifier training}

Training cohort

Fifty confirmed gBRCA1-mutated (m), 10 confirmed gBRCA2 m cases, and 13 patients without a family history of ovarian or breast cancer (controls) were identified through the Netherlands Cancer Institute (NKI, Amsterdam, the Netherlands) tumor registration database and the Erasmus Medical Center (see Table 1). This study was conducted in concordance with Dutch law and national guidelines that allow for the analysis of residual tissue specimens obtained for diagnostic purposes and anonymized publication of the results (38).

\section{DNA isolation and BRCA1 methylation assay}

Histologic classification and grading, according to the two-tiered system, were performed by CHMvD and PCEG. We isolated DNA from formalin-fixed paraffin-embedded (FFPE) tumor slides, using (micro)dissection to obtain 60\% tumor percentage with the Qiagen DNA mini kit (Qiagen, catalog no. 51306) and for BRCA1 methylation, using an MLPA kit (MRC-Holland) as described before (30), see Supplementary Materials and Methods for extended description.

\section{NimbleGen 135K oligonucleotide array comparative genomic hybridization}

We obtained NimbleGen $135 \mathrm{~K}$ array comparative genomic hybridization $(\mathrm{aCGH})$ profiles from the training set samples. The $135 \mathrm{~K}$ array data were mapped to the $\mathrm{BAC} \mathrm{aCGH}$ platform for dimension and noise reduction by averaging probes covered by the BAC clone, as described before $(39,40)$. The data discussed in this publication have been deposited in NCBI's Gene Expression Omnibus (GEO) and are accessible through GEO Series accession number GSE111688 (https://www.ncbi.nlm.nih.gov/geo/query/acc.cgi?acc=GSE111688).

Table 1. Pathologic characteristics of samples within the training set.

\begin{tabular}{lllll}
\hline & & Control & gBRCA1 & gBRCA2 \\
\hline Subtype & Serous & 13 & 48 & 10 \\
& Endometrial & 0 & 1 & 0 \\
\multirow{4}{*}{ Grade } & Other & 0 & 1 & 0 \\
& Low grade & 1 & 1 & 0 \\
& High grade & 12 & 48 & 10 \\
& Missing & 0 & 1 & 0 \\
\hline
\end{tabular}


Estimating the performance of the classifier in a 10-fold doubleloop cross-validation loop

We carried out nested double-loop 10-fold cross-validation of shrunken centroid classifiers to classify ovarian cancer as being similar to gBRCA1 m (BRCA1-like) or gBRCA2 m (BRCA2-like) or controls (C; ref. 29). We obtained the delta threshold by optimizing the classification error and selecting the sparsest model within one SE of the optimal solution (41). Subsequently, we used the model at the selected threshold to predict the samples in the outer loop. The AUC of the receiver/operator curve (ROC) of our predictions of the class labels is computed using samples that are left out of the training procedure. After estimating this unbiased performance, we trained the full dataset's final model using the inner loop. See Supplementary Materials and Methods for pseudocode.

\section{Validation in TCGA dataset}

We downloaded the Infinium HumanMethylation27 BeadChip methylation, segmented genome-wide human SNP6.0 copy number, and gene expression data from the firebrowse.org archives of the ovarian cancer TCGA data (version 2015082100.0.0). BRCA1 and $B R C A 2$ mutation status were obtained from cBioPortal, which stores the somatic and germline mutation status used in the original TCGA articles (42). The BRCA1 promoter methylation status was obtained by correlating the methylation and gene expression data. We used methylation probe cg10893007 because of the strongest Pearson correlation with $\log B R C A 1$ gene expression ratio among the $B R C A 1$ promoter probes and close location to the MLPA probe used in the previous study (30) among those covering the BRCA1 promoter in TCGA dataset.

\section{Classifying TCGA data}

TCGA data were mapped to the NKI BAC array CGH positions, as described before (39). In short, SNP6.0 probes within the start and end positions of the BAC clone were averaged (mapped TCGA). We adjusted for differences in scaling and centering by using a method similar to quantile normalization. Briefly, we performed linear regression by fitting a generalized linear Gaussian model with identity link function to the sorted location-wise average DNA copy-number values of the NimbleGen data and the sorted location-wise DNA copynumber averages of the mapped TGCA dataset. Subsequently, we used the obtained alpha coefficient to correct the centering and the obtained beta coefficient to correct the scaling of the mapped TCGA data, followed by classification. We validated this method on samples that had been analyzed before (39) both on NimbleGen $135 \mathrm{~K}$ aCGH and SNP6.0. See Supplementary Materials and Methods for additional information. Subsequently, samples were classified as $B R C A$-like if the predicted probability was $>0.5$ and non-BRCA-like if the predicted probability was $\leq 0.5$, as was predefined in the training set.

\section{Performance in TCGA data}

The sensitivity and specificity of detecting the class labels gBRCA1/ BRCA2 mutation, sBRCA1/BRCA2 mutation, and BRCA1 promoter hypermethylation were calculated. The Youden index, (sensitivity + specificity) -1 , was used as a balanced measure to assess performance. This readout resembles the equal weighting of sensitivity and specificity in the training process.

\section{Independent validation within AGO-TR1 cohort study}

Within the consecutive cohort study AGO-TR1 (NCT02222883), 523 patients with ovarian cancer were counseled and enrolled in 20 AGO study group centers in Germany. The ethical committee approved the study protocol of the Landesaerztekammer Nordrhein (Nr. 2014340). Written informed consent was obtained before any study-related procedure. All individuals were 18 years or older and displayed a primary (PR: $n=281$ ) or platinum-sensitive relapsed (RE: $n=242$ ) ovarian cancer, defined as relapse after a platinum-free interval of at least 6 months. The AGO study group documented clinical data including demographics, medical and family history as well as disease characteristics.

The Center of Hereditary Breast and Ovarian Cancer in Cologne performed the genetic analyses on blood samples of all participants and FFPE tumor samples of 496 patients as described previously $(36,37)$. In short, a paired multi-gene panel sequencing of germline and tumor samples analyzing 27 ovarian cancer-related and DNA repair genes (see Supplementary Materials and Methods) was performed with a complementary use of copy-number variation analysis for the detection of large genomic rearrangements in germline samples. All variants were classified using a five-tier variant classification system as proposed by the International Agency for Research on Cancer Unclassified Genetic Variants Working Group, namely, deleterious = class 5, likely deleterious $=$ class 4 , variant of uncertain significance $=$ class 3 , likely benign $=$ class 2 and benign $=$ class 1 . For somatic variants, the My Cancer Genome database (http://www.mycancergenome.org), the IARC TP53 database (https://p53.iarc.fr), and the ClinVar database (https://www.ncbi.nlm.nih.gov/clinvar/) were also considered for variant classification. Variants reported to occur in large outbred control reference groups at an allele frequency of $>1 \%$ were generally considered benign. Class $4 / 5$ variants were subsequently defined as "deleterious variants." Variants were considered somatic if they were not identified in a paired germline analysis of the corresponding blood sample. Also, quantitative methylation assays analyzing BRCA1, $P A L B 2$, and $R A D 51 C$ promoter regions were carried out as described before (37). In total, the complete data of 473 individuals were successfully generated (ref. 37; see CONSORT-like flow diagram; Supplementary Fig. S1).

\section{Selection of the study sample}

We selected 300 samples with matching germline and somatic mutation status and successful performed methylation analyses for BRCA-like classification (see Table 2), including all available samples with deleterious germline and somatic variants (IARC class $4 / 5$ ) in $B R C A 1 / 2(n=118)$, other HR-related and hereditary non-polyposis colorectal cancer (HNPCC) genes $(n=44)$, and somatic (s) BRCA1/2VUS (variants of unknown significance; $n=4$ ). As controls, we randomly selected a similarly sized group $(n=134)$ from the rest of the main cohort taking PR/RE status, age at diagnosis, and sTP53 mutations into account. Samples with only gBRCA1/2 VUS $(n=15)$ were excluded.

\section{DNA isolation}

Hematoxylin and eosin-stained $3 \mu \mathrm{m}$ tissue sections were centrally investigated (Institute of Pathology, University Hospital Bonn, Bonn, Germany). Tumor areas containing $>80 \%$ tumor nuclei were chosen and dissected for DNA isolation. DNA isolation from FFPE tumor samples was conducted using standard procedures, as described previously (37).

\section{Low-coverage whole-genome sequencing and BRCA1/2-like classification}

Low-coverage whole-genome sequencing (lcWGS) was centrally performed (NKI, Amsterdam, the Netherlands), as described earlier (39). Library preparation was performed with an input of $200 \mathrm{ng}$ 
Schouten et al.

Table 2. Occurrence of genetic and clinical features within the samples of the presented study cohort ${ }^{\mathrm{a}}$.

\begin{tabular}{|c|c|c|c|c|c|}
\hline & & \multicolumn{2}{|c|}{ A } & \multicolumn{2}{|l|}{ B } \\
\hline & & $\begin{array}{l}\text { Study } \\
\text { cohort }(n)\end{array}$ & [\%] & $\begin{array}{l}\text { Study cohort }(n) \text { without } \\
B R C A / \text { HR gene mutations }\end{array}$ & [\%] \\
\hline Germline and somatic & gBRCA1 & 69 & 23.0 & & \\
\hline \multirow{7}{*}{ Mutation status } & gBRCA2 & 23 & 7.7 & & \\
\hline & gHR genes & 36 & 12.0 & & \\
\hline & $\mathrm{SBRCA1/2}$ & 28 & 9.3 & & \\
\hline & sHR genes & 12 & 4.0 & & \\
\hline & gHNPCC genes & 3 & 1.0 & 3 & 2.1 \\
\hline & sTP53 & 251 & 83.7 & 106 & 75.2 \\
\hline & SPTEN/PIK3CA & 17 & 5.7 & 8 & 5.7 \\
\hline \multirow[t]{2}{*}{ Methylation status } & BRCA1 methyl & 28 & 9.3 & 26 & 18.4 \\
\hline & RAD51C methyl & 4 & 1.3 & 2 & 1.4 \\
\hline \multirow[t]{8}{*}{ Histologic subtype } & High-grade serous & 246 & 82.0 & 110 & 78.0 \\
\hline & High-grade endometrioid & 16 & 5.3 & 9 & 6.4 \\
\hline & Serous, grade unknown & 6 & 2.0 & 3 & 2.1 \\
\hline & Low-grade serous & 7 & 2.3 & 4 & 2.8 \\
\hline & Low-grade endometrioid & 4 & 1.3 & 1 & 0.7 \\
\hline & Clear cell & 2 & 0.7 & 2 & 1.4 \\
\hline & Mucinous & 4 & 1.3 & 3 & 2.1 \\
\hline & Other/missing & 15 & 5.0 & 9 & 6.4 \\
\hline \multirow[t]{3}{*}{ Clinical features } & PR & 160 & 53.3 & 83 & 58.9 \\
\hline & $\mathrm{RE}$ & 140 & 46.7 & 58 & 41.1 \\
\hline & Total & 300 & & 141 & \\
\hline
\end{tabular}

Note: Genetic and epigenetic features coincided within some samples and were listed in each subcategory concerned. The mean age at diagnosis was 57.6 years (range, 18-83 years) in the main cohort and 59.4 years (range, 21-83 years) excluding all BRCA1/2-mutated or HR gene-mutated cases.

Abbreviations: PR, primary ovarian cancer; RE, recurrent disease.

${ }^{a} \mathrm{~A}$ lists all samples, whereas $\mathrm{B}$ excludes all samples with a $B R C A$ or other HR gene mutation, as those were enriched within the study cohort.

double-stranded DNA derived from FFPE-tumor samples using the TruSeqDNA LT Sample Preparation kit (Illumina). Ten explicit indexed samples were equimolarly pooled and sequenced in one lane on an Illumina HiSeq 2500 device (Illumina). Single-read sequencing (read-length $65 \mathrm{bp}$ ) was performed with an aimed coverage of $0.5 \times$ and sequences were aligned to reference genome GRCh38. Reads were counted in $20 \mathrm{~kb}$ non-overlapping bins, corrected for CG bias and corrected for local alignment-bases estimated mappability, resulting in ${ }^{2} \log$ count ratios.

The $20 \mathrm{~kb}$ resolution ${ }^{2} \log$ ratios were mapped to the $1 \mathrm{MB}$ resolution input for the classifier (mapped AGO-TR1). This was done by averaging the ${ }^{2} \log$ count ratios within the $1 \mathrm{MB}$ bins (surrounding the BAC clone locations of the BAC platform). Because the classifier's training was performed on oligonucleotide array CGH data, we performed a correction of centering and scaling of the data with the next-generation sequencing (NGS) platform in this study. This correction is akin to quantile normalization and was performed by fitting a linear regression model with Gaussian distribution and identity link function using the $\mathrm{R}$ glm function to the sorted location-wise average of the training set and the mapped AGOTR1 dataset. Subsequently, we used the obtained alpha coefficient to correct the centering and the obtained beta coefficient to correct the scaling of the new data (Supplementary Materials and Methods). We validated this method on samples that had been analyzed both on NimbleGen $135 \mathrm{~K}$ array CGH and NGS in the cross-platform robustness dataset we described previously (39). Subsequently, samples were classified as BRCA-like if the predicted probability was $>0.5$ and non- $B R C A$-like if the predicted probability was $\leq 0.5$ as was predefined in the training set.

\section{Statistical analyses}

Fisher exact and $\chi^{2}$ test were applied, where appropriate, to calculate the level of significance. All tests were two sided, and a $P<0.05$ after correction for multiple testing using the Benjamini-Hochberg approach was considered significant.

\section{Results}

\section{Classifier training}

Description of the cohort

We generated copy-number profiles of 13 control, $50 \mathrm{gBRCA} 1 \mathrm{~m}$, and $10 \mathrm{gBRCA} 2 \mathrm{~m}$ ovarian cancer, and show the pathologic characteristics of these tumors in Table 1. Figure 1 presents the average profiles of $\mathrm{gBRCA} 1 \mathrm{~m}$ and $\mathrm{g} B R C A 2 \mathrm{~m}$ versus control ovarian cancers. Frequent genomic losses and the patterns of copy-number aberration in our dataset are similar to other datasets, for example, the shape and occurrence of the gain on chromosome $8 \mathrm{q}$, the loss on $4 \mathrm{q}$, and the loss of chromosome X $(43,44)$.

\section{Training ovarian cancer-specific classifiers}

First, we classified the ovarian cancer copy-number profiles with the breast cancer BRCA1-like and BRCA2-like classifiers $(30,31)$ and obtained an AUC of 0.51 [95\% confidence interval (CI): 0.4-0.63] for 


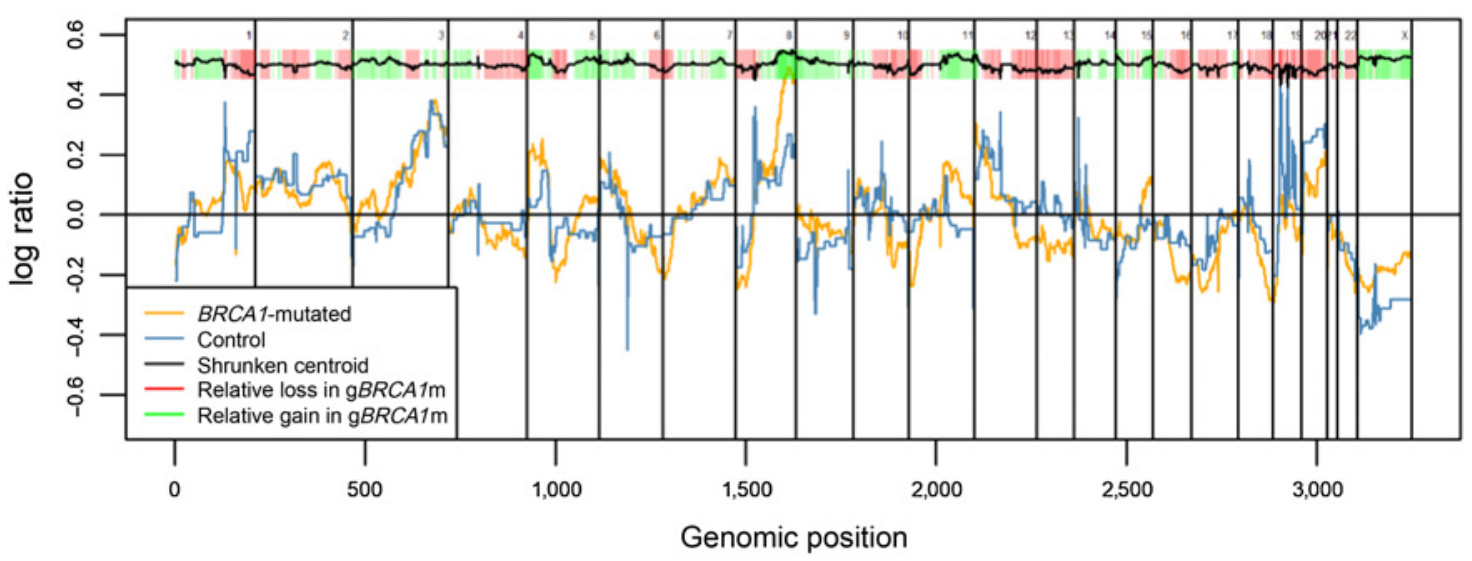

B BRCA2m vs. control average profiles and shrunken centroids

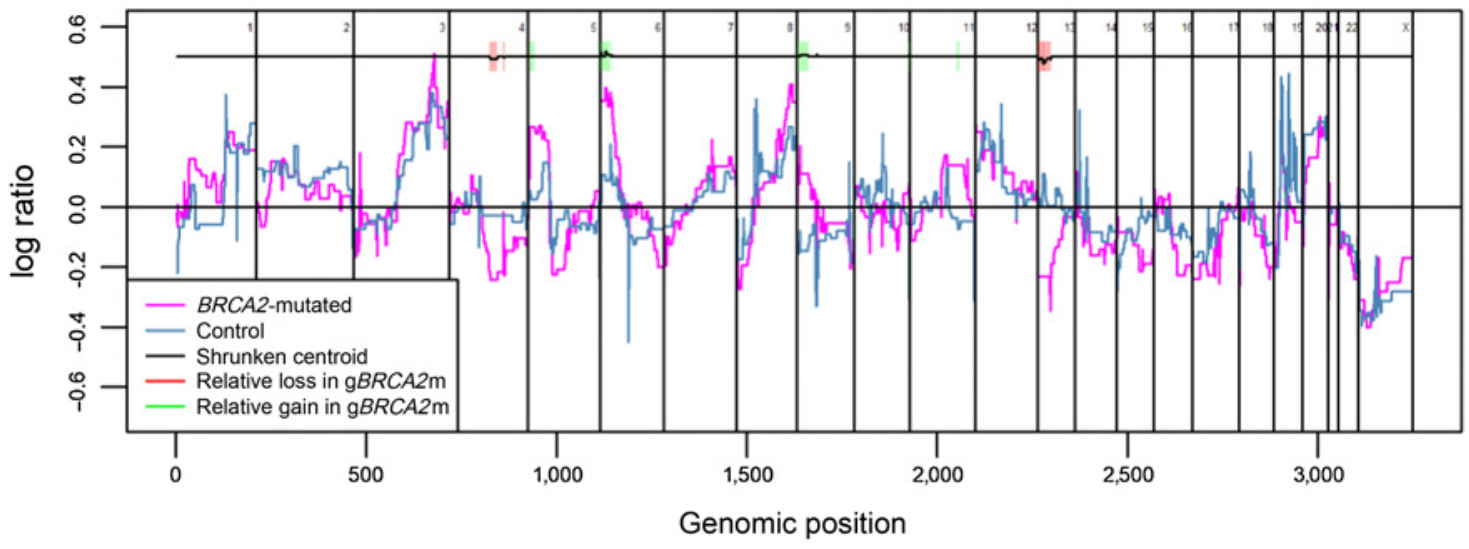

Figure 1.

Average copy-number aberration profiles of $B R C A 1$ - and $B R C A 2$-mutated ovarian cancer. A, Average copy-number aberration profile of $50 \mathrm{~g} B R C A 1 \mathrm{~m}$ ovarian cancers and 13 control ovarian cancers. On the $x$-axis, the cumulative genomic position, in megabases, and the $y$-axis, the average ${ }^{2}$ log ratio of tumor over normal DNA. B, Average copy-number aberration profile of $10 \mathrm{gBRCA} 2 \mathrm{~m}$ ovarian cancers and 13 control ovarian cancers. On the $x$-axis, the genomic position and on the $y$ axis the average ${ }^{2} \log$ ratio of tumor DNA over normal DNA.

gBRCA1 m and an AUC of 0.63 (95\% CI: 0.28-0.98) for gBRCA2 m ovarian cancer in the training set. Given this low performance, we trained shrunken centroid classifiers on ovarian cancer data to investigate whether the performance could be improved. These shrunken centroids select genomic regions and weights that are discriminative for the BRCA-like and non-BRCA-like class. We used ten-fold crossvalidation and trained on the class labels gBRCA1 $\mathrm{m}$ versus control and gBRCA2 $\mathrm{m}$ versus control. We observed a cross-validated AUC of 0.67 $(0.55-0.78)$ and $0.91(0.79-1)$, respectively, for BRCA1-like and BRCA2-like classification (Table 3).

Because the NimbleGen aCGH platform is not available anymore, we validated cross-platform compatibility of our classifier using the same methods and dataset as described previously (39); see Supplementary Materials and Methods.

External validation of ovarian cancer BRCA classifiers in TCGA data

We used TCGA ovarian cancer data as an external validation set for our newly trained ovarian cancer classifiers. We classified 358 of 583 samples as having a BRCA1-like profile. Within these 358, 25/
26 gBRCA1, 10/11 somatic BRCA1-mutated tumors, and 67/69 $B R C A 1$-methylated tumors were classified as BRCA1-like, resulting in an overall sensitivity of $96.2 \%$ (95\% CI: $0.90-0.99$ ). Specificity, however, is lower, at $40 \%$ (95\% CI: $0.36-0.45)$. 350 of 583 samples were assigned to be BRCA2-like. Within these $350,20 / 25 \mathrm{gBRCA} 2 \mathrm{~m}$ and $8 /$ $11 \mathrm{~s} B R C A 2 \mathrm{~m}$ samples were classified as BRCA2-like, resulting in a sensitivity of $77 \%$ (95\% CI: $0.6-0.9$ ) and a specificity of $41 \%$ (95\% CI: $0.37-0.49)$.

\section{Validation within the AGO-TR1 cohort}

We further analyzed both the germline/somatic mutation status of $B R C A 1 / 2$ and BRCA1 promoter hypermethylation in the AGO TR1 study. In addition, we aimed to characterize those patients called $B R C A$-like without a mutation in BRCA1 or BRCA2.

\section{Study sample}

In total, 300 samples with complete genetic and epigenetic information available, were analyzed. A total of 130 germline and 43 somatic deleterious variants in BRCA1/2 and other HR genes were present in 159 ovarian cancer samples (see Table 2). The majority 
Table 3. Cross-validated results of training shrunken centroids classifiers to distinguish BRCAT- or BRCA2-mutated from control ovarian cancers.

\begin{tabular}{|c|c|c|c|c|c|c|c|}
\hline & AUC $(95 \% \mathrm{Cl})$ & $\begin{array}{l}\text { Sensitivity } \\
(95 \% \mathrm{CI})\end{array}$ & $\begin{array}{l}\text { Specificity } \\
(95 \% \mathrm{CI})\end{array}$ & & Control & BRCA1m & $B R C A 2 \mathrm{~m}$ \\
\hline $\mathrm{g} B R C A 1$ & 0.67 & 0.76 & 0.69 & Predicted control & 9 & 12 & 0 \\
\hline vs. control & $(0.55-0.78)$ & $(0.62-0.87)$ & $(0.39-0.91)$ & Predicted BRCA1 & 4 & 38 & 0 \\
\hline$\overline{\mathrm{g} B R C A 2}$ & 0.91 & 0.90 & 0.92 & Predicted control & 12 & 0 & 1 \\
\hline vs. control & $(0.79-1)$ & $(0.55-1)$ & $(0.64-1)$ & Predicted $B R C A 2$ & 1 & 0 & 9 \\
\hline
\end{tabular}

Abbreviations: AUC, area under the curve of the receiver operating characteristic; $\mathrm{Cl}$, confidence interval.

displayed a $B R C A 1$ mutation (g: $n=69$, s: $n=17)$, a BRCA2 mutation (g: $n=23$, s: $n=11$ ), or a RAD51C mutation (g: $n=13$ ). One of these samples presented a germline double mutation of gBRCA1 and gBRCA2 and was analyzed with the gBRCA1 m group; one somatic $B R C A 2$ mutation coincided with a $\mathrm{g} B R C A 2$ mutation and was assigned to the gBRCA2 group. Seven other samples with a $B R C A$ mutation (g: $n=5, \mathrm{~s}: n=2$ ) displayed a class $4 / 5$ in another HR gene as well and were analyzed with the respective $B R C A$-mutated group.

Most ovarian cancers presented with high-grade serous histology (82\%), and a tumor TP53 mutation was found in $83.7 \%$. Promoter hypermethylation was detected in $9.3 \%$ of samples for BRCA1 $(n=28)$ and in $1.3 \%$ of samples $(n=4)$ for RAD51C. The mean age at diagnosis was 57.6 years, and 160 primary ovarian cancer and 140 platinumsensitive recurrent cases were included.

A total of 223 tumors showed a BRCA1-like or BRCA2-like classification $(74.3 \%)$. Seventy-seven ovarian cancer thereof were only BRCA1-like, 26 were only BRCA2-like, and 120 were both $B R C A 1$ - and BRCA2-like.

\section{Detection of BRCA-associated ovarian cancers with BRCA-like} classification

As there is no established gold standard to define BRCAness in general, we first focused on the detection rate of tumors derived from deleterious germline mutations in BRCA1/2 (see Table 4; Fig. 2). 67 of 69 BRCA1-mutated cases had a BRCA1-like (detection rate of $97.1 \%$ ), whereas 16 of 22 samples with a $B R C A 2$ class $4 / 5$ variant in germline were BRCA2-like (detection rate of $72.7 \%$ ). All remaining BRCA2associated cases were identified by combining both classifiers, that is, being $B R C A 1$ - and/or BRCA2-like.

Regarding deleterious somatic variants, 15 of 17 (88.2\%) samples with a BRCA1 mutation were identified by the BRCA1-like classifier. As the other two tumors displayed a BRCA2-like phenotype, both classifiers' application detected $100 \%$ of the mutated cases. The $B R C A 2$-like classifier confirmed 6 of $10 \mathrm{~s} B R C A 2 \mathrm{~m}$ cases. The $B R C A 1$-like classifier found no additional sample.

Of the 28 examined samples with a BRCA1 promoter hypermethylation, 27 displayed a $B R C A 1$-like profile (96.4\%), one was classified as non-BRCA-like.

To better understand the reason for the non-BRCA-like classification of the samples mentioned above, we took a closer look at their molecular genetic background (see Supplementary Table S1).

Within the gBRCA1 $\mathrm{m}$ cohort, the non-BRCA-like tumors harbored LOF (loss of function) mutations. Both tumors did not show a relevant elevation of variant allele frequency (VAF) of the deleterious $B R C A 1$ variant in the tumor than germline and none or only a low frequent sTP53 mutation. One of these BRCA1 variants was found in two other samples, which were classified as BRCA1and BRCA2-like.
Regarding the sBRCA2 $\mathrm{m}$ samples, two of the four non- $B R C A$ like cases displayed several deleterious class $4 / 5$ variants in other analyzed genes, next to the sBRCA2 mutation with a VAF of $10 \%$, respectively, $12 \%$, but no STP53 mutation. Within the BRCA1methylated tumor, no further deleterious somatic alteration was found.

Classification of samples with HR gene alterations beyond BRCA1/2 and mutations in HNPCC genes

Alterations in further HR genes (ATM, BARD1, BRIP1, CHEK1, CHEK2, FAM175A, FANCM, MRE11A, NBN, PALB2, RAD50, $R A D 51 C, R A D 51 D, X R C C 2$ ) varied in being classified as BRCA-like (see Supplementary Table S2).

Table 4. Detection rate of the $B R C A 7-(A)$ and $B R C A 2$-like classifier (B) in $B R C A$-associated cancers. (C) shows the complementary application of both classifiers; BRCA1- and/or BRCA2-like.

\begin{tabular}{llll}
\hline A) & BRCA1-like & [\%] & Total \\
\hline gBRCA1 & 67 & 97.1 & 69 \\
SBRCA1 & 15 & 88.2 & 17 \\
BRCA1 methyl & 27 & 96.4 & 28 \\
BRCA1 WT & 88 & 47.3 & 186 \\
Total & 197 & 65.7 & 300 \\
\hline B) & BRCA2-like & [\%] & Total \\
\hline gBRCA2 & 16 & 72.7 & 22 \\
SBRCA2 & 6 & 60.0 & 10 \\
BRCA2 WT & 124 & 46.3 & 268 \\
Total & 146 & 48.7 & 300 \\
\hline C) & & & \\
& BRCA1/2-like & {$[\%]$} & Total \\
\hline gBRCA1 & 67 & 97.1 & 69 \\
gBRCA2 & 22 & 100.0 & 22 \\
SBRCA1 & 17 & 100.0 & 17 \\
sBRCA2 & $6^{\text {a }}$ & 60.0 & $10^{\text {a }}$ \\
BRCA1 methyl & $27^{\text {a }}$ & 96.4 & $28^{\text {a }}$ \\
BRCA WT & 85 & 54.8 & 155 \\
Total & 223 & 74.3 & 300 \\
\hline
\end{tabular}

Abbreviations: g, germline; s, somatic; methyl, promoter hypermethylation; WT, wild type.

ane sample displayed both a somatic BRCA2 mutation and a BRCA1 methylation and was classified as BRCA1- and BRCA2-like. Therefore, it was presented in both groups but counted only once for the total sum.

The sample displaying both a gBRCA7 and 2 mutation was counted among the gBRCA1 samples; the sample with a BRCA2 mutation in germline and tumor was counted among the gBRCA2 samples. 
Figure 2.

$B R C A$-like phenotype according to molecular genetic background. Each bar shows the distribution by percentage ( $y$-axis) of BRCAT- and BRCA2-like, only $B R C A 7$-like, only $B R C A 2$-like, and non-BRCA-like profiles within the presented molecular genetic subgroup ( $x$-axis). methyl, promoter hypermethylation; WT, wild type.

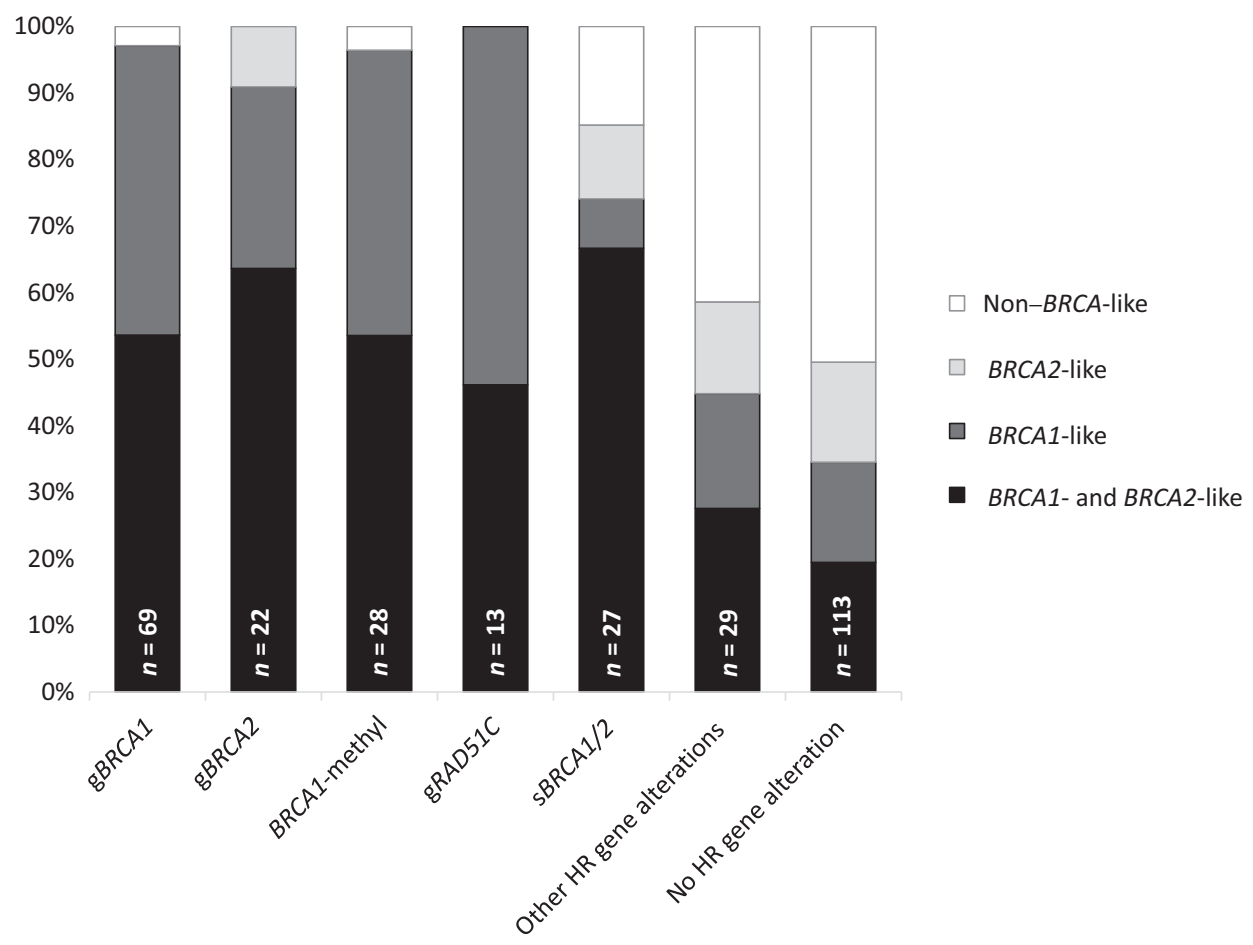

Within the study cohort, 44 tumors without $B R C A$ mutation or $B R C A 1$ methylation displayed a genetic alteration in another ovarian cancer risk- or HR gene (except sTP53 mutations), of which 30 were classified as BRCA-like (68.2\%; only BRCA1-like $n=11$, only BRCA2like $n=4, B R C A 1$ - and BRCA2-like $n=15$ ).

A distinct statistical association was observed for the largest subgroup, the $R A D 51 C$-associated tumors. All germline mutation-carriers developed a BRCA-like ovarian cancer (only BRCA1-like $n=6$, $B R C A 1$ - and BRCA2-like $n=7$ ).

No clear statistical association could be observed for most other HR gene mutated samples and samples with $R A D 51 C$ methylation (see Supplementary Table S2; Fig. 2) possibly due to small numbers and coinciding aberrations.

Samples with a class 4/5 variant in $R A D 51 D$ (g: $n=3$, s: $n=1$ ) presented a BRCA1- and BRCA2-like profile in three of four cases and FANCM-associated ovarian cancer (g: $n=2$, s: $n=3$ ) in three of five samples, one sample further presented with only a BRCA1-like phenotype. All four ATM-associated samples showed a BRCA-like profile.

Three of six PALB2-associated cancers presented a BRCA-like profile, including two samples with each an $A T M$ mutation (see above) and a BRCA1 methylation. Therefore, the underlying mechanism leading to a BRCA-like phenotype was unclear.

Samples with each a mutation in CHEK1 (g), FAM175A (g), and $B A R D 1$ (s) as well as three tumors with a germline mutation in HNPCC genes ( $M S H 2 n=2$, MSH6 with additional somatic MSH6and BRIP1 mutation, $n=1$ ) were examined and showed no BRCA-like profile.

Epigenetic alterations in $R A D 51 C$ were rare. Four samples with a $R A D 51 C$ promoter hypermethylation were analyzed, of which one was derived from a germline BRCA1 mutation carrier, presenting a $B R C A 1$ - and BRCA2-like profile, assumingly due to the known mutation. Of the three remaining samples, one was classified as BRCA1- and BRCA2-like; the other two (one of them from a gMRE11A mutation carrier) displayed a non-BRCA-like phenotype.
Molecular genetic background of $B R C A$-like samples

Within the cohort, 223 samples displayed a BRCA1/2-like phenotype. These profiles were associated with a germline mutation in $B R C A 1 / 2$ in 89 and a somatic mutation in $B R C A 1 / 2$, respectively a $B R C A 1$ promoter hypermethylation in 49 of these samples. In another 29 different samples, a somatic or germline class $4 / 5$ variant in another HR gene (esp. $R A D 51 C$ ) or a $R A D 51 C$ promoter hypermethylation can explain the presence of a BRCA-like phenotype.

For the remaining 55 BRCA1/2-like samples (24.6\%), no aberration affecting the HR could be detected by gene panel or methylation analyses (see Fig. 3). Regarding all ovarian cancer without a genetic or epigenetic alteration in BRCA1/2 $(n=155), 54.8 \%(n=85)$ display a $B R C A 1 / 2$-like phenotype. Excluding all HR gene-mutated cases ( $n=$ $113)$, as the study cohort was enriched for those, the BRCA1/2-like rate was $48.7 \%(n=55)$.

Furthermore, a BRCA-like phenotype was correlated with highgrade serous histology, sTP53 mutations and could be observed tendentially more often, when the patient was included with platinum-sensitive recurring disease than at primary diagnosis. A non$B R C A$-like profile was seen in association with low-grade serous histology, in combination with PTEN or PIK3CA mutations and with gHNPCC mutations (see Table 5).

\section{Discussion}

In this study, we identified BRCA1-like and BRCA2-like copynumber classifiers in analogy to those we previously trained in breast cancer $(30,31)$. Furthermore, we showed in an independent cohort that these signatures identify patients with germline and somatic mutations and promoter hypermethylation in BRCA1/2. In addition, we were able to investigate underlying molecular mechanisms in $B R C A$-like cases without a deleterious variant in BRCA1 or BRCA2.

Our approach is supported by the analogy to breast cancer $B R C A$ like classifiers. We previously showed that these assays predicted both 


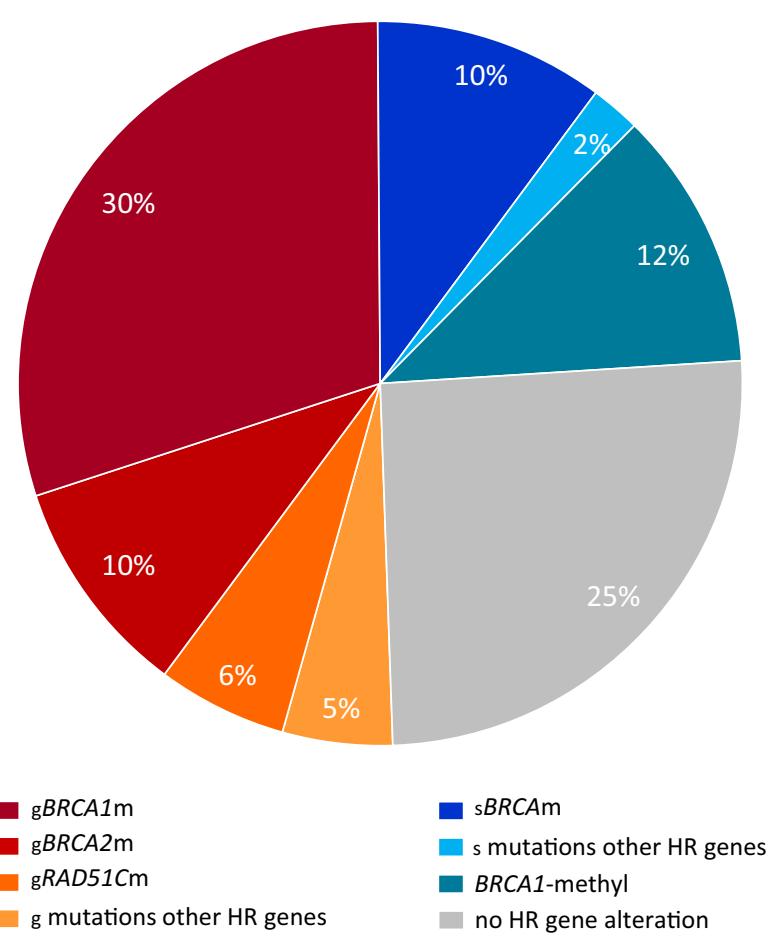

Figure 3.

Molecular genetic background of BRCA-like cases. A total of 223 samples are classified as BRCA1- and/or BRCA2-like. The pie chart shows the portion of samples with a specified (epi-)genetic alteration of a gene, involved in homologous recombination in this subgroup. In $25 \%$ of the cases, no HR-affecting variant was detected by NGS or methylation analyses. methyl, promoter hypermethylation.

mutation status and sensitivity to DNA-damaging agents $(28,30,31)$. We hypothesized that in ovarian cancer, with the same genetic driver, a similar signature would arise. Applying the breast cancer classifiers in ovarian cancer, we observed that those did not predict mutation status sufficiently. Therefore, we set out to train ovarian cancer-specific classifiers that we validated as being robust with an array- and sequencing-based input data.

Another advantage of this study is that after initial promising results within cross-validation of the training set and in TCGA test dataset were obtained, the AGO-TR1 study provided a large and wellcharacterized cohort in which we could validate the prediction of mutation status.

Overall, the BRCA1-like classifier showed a convincing performance with detecting BRCA1-mutated and BRCA1-methylated cancers in more than $90 \%$ of the cases. When applying both classifiers in the AGO-TR1 trial, more than $95 \%$ of all BRCA1/2-associated cancers in the cohort were detected. Extensive germline and tumor genetic analysis provided additional support for the BRCA-like class showing alterations in $B R C A 1 / 2$ or other HR genes in $75 \%$ of cases.

Germline RAD51C mutations demonstrated a clear association with a BRCA1-like profile. For most other HR gene mutations, no such correlation could be proven, possibly due to low sample size or because hereditary predisposition does not always lead to a tumor with HRD (45), stressing the need for complementary germline/tumor analyses to address therapeutic relevance as well as hereditary predisposition.
We detected a BRCA-like phenotype in three-quarters of the AGOTR1 ovarian cancer samples. This is more than in the recently published randomized controlled trials PAOLA-1 (26) and PRIMA (25), where the rate was about half, and might lead to suspicion of overcalling in the current study. However, the current substudy applied skewed selection criteria, so most cases with a BRCA1/2 mutation $(118 / 126 ; 93.7 \%)$ or a mutation in one of the other HR genes $(41 / 42 ; 97.6 \%)$ were analyzed by lcWGS and only around $50 \%$ of the cases without BRCA- or HR-related mutation. If we would extrapolate the findings in the selected population to the full AGO TR1 trial, the proportions are entirely in line with other trials $(14,24-26)$. Regarding the percentage of primary HGSOC responding to platinum compounds of $80 \%$ or above, the HRdeficient population in ovarian cancer might even be within those ranges. Yet, this remains to be investigated in a study for predictive value.

Another point of attention is the possible failure to detect BRCA1associated tumors despite the detection rate of over $90 \%$. One reason for this might be the contamination of normal tissue within the sample, which might have been the case with two non-BRCA-like sBRCA2mutated tumors and the two gBRCA1-mutated tumors (see Supplementary Table S1), which also displayed a noisy profile (see Supplementary Materials and Methods). However, displaying a non-BRCAlike profile when carrying a deleterious $B R C A$ mutation could also be the correct interpretation, for example, if no second hit/LOH is observed, in which the patient might have developed a sporadic, HR-proficient tumor in the presence of a germline mutation (45).

The BRCA2-like classifier was less predictive of $B R C A / H R$ gene mutation status, which might be caused by the smaller training set of $\mathrm{gBRCA2}$-mutated samples. As this classifier alone is insufficient to predict $B R C A 2$ mutation status, training a classifier with a more extensive set of gBRCA2-mutated samples might be required. In its current state, it only serves to detect samples with a gBRCA2 mutation and $s B R C A 1 / 2$ mutations missed by the BRCA1-like classifier.

In the literature, several other assays have been described measuring HRD in ovarian cancer $(15,17,21,46)$, some of which are also partly based on derivatives of SNP/copy-number profiles. Our approach differs from other tests because it uses genomic location-specific aberrations. This could lead to additional information being retained, such as aberrations that might collaborate with the underlying HRD mechanism. In addition, the presented classifiers are tissue specific, which might be an advantage compared with other HRD assays, as a response to targeted strategies can depend on the tissue of origin. An example of this phenomenon is the effect of $\mathrm{BRAF}(\mathrm{V} 600 \mathrm{E})$ inhibition of $B R A F(\mathrm{~V} 600 \mathrm{E})$-mutant cancer in melanoma versus colon cancer (47). Copy-number profiles are generally considered static, lowresolution snapshots. Mutational signatures derived from deeper more in-depth sequencing techniques might identify more directly the traces of errors fixed in the absence of HR and lead to more specific profiles than copy-number profiling $(18,48)$. While signatures better describe the history of HRD (49), tests like the RAD51 foci formation assay might yield direct information on the functionality of the HR pathway (50). Furthermore, an analysis in the therapeutic setting, preferably direct comparison of different assays, is mandatory to identify those with the best discriminatory power to predict HRD-specific therapy. For clinical application, availability, adaptability, and costeffectiveness should be taken into account.

A limitation of the current study is that the gold standard for true HR deficiency should be determined with therapy response, which was unavailable. Although the recognition of mutations in BRCA1/2 and 


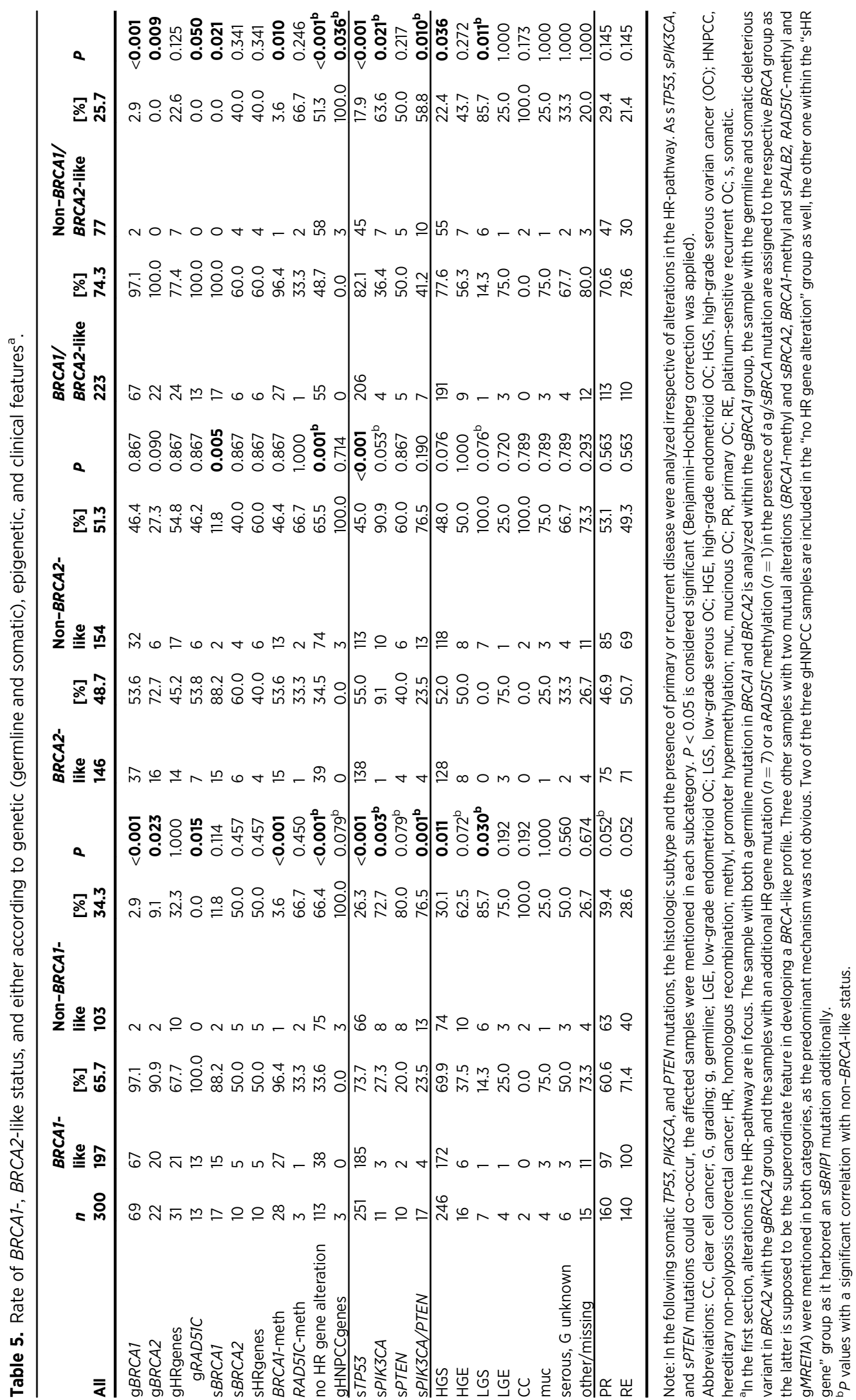


other HR genes that might explain the BRCA-like signature is promising, follow-up studies should be done to determine predictive value.

In conclusion, we have shown a convincing performance of the BRCA1-like classifier and the combination of both classifiers in detecting $B R C A$ mutations/methylation. In addition, around half of the non- $B R C A$-associated ovarian cancer cases displayed a $B R C A$-like phenotype as well. Therefore, it would be interesting to investigate, if the classifiers could also serve as predictive markers of HRD-directed therapy like PARPi, which has to be evaluated in subsequent clinical studies.

\section{Authors' Disclosures}

P.C. Schouten reports a patent for BRCA-like classifier in ovarian cancer pending and partner is employed by AstraZeneca. L. Richters reports grants from AGO Research $\mathrm{GmbH}$ during the conduct of the study; in addition, L. Richters has a patent for BRCA1/2-like classifier pending and was supported by the program for Temporary Substitutes for Clinicians (Gerok substitute positions) at the Faculty of Medicine, University of Cologne. C. Ernst reports grants from AGO Research GmbH during the conduct of the study; in addition, C. Ernst has a patent for BRCA1/2 classifier pending. F. Marmé reports other support from AGO Research GmbH during the conduct of the study as well as personal fees from Roche, AstraZeneca, GSK/Tesaro, PharmaMar, Amgen, GenomicHealth, CureVac, EISAI, Clovis, JanssenCilag, Immunomedics, MSD, Pfizer, Lilly, Novartis, Agendia, Pierre-Fabre, Gilead, and SeaGen outside the submitted work. S. Schmidt reports grants from AGO Studiengruppe $\mathrm{GmbH}$ during the conduct of the study. K. Prieske reports personal fees from GSK, AstraZeneca, Roche, Clovis Oncology, and Molecular health outside the submitted work. N. de Gregorio reports personal fees and nonfinancial support from Roche and personal fees from AstraZeneca, GSK, MSD, and Clovis outside the submitted work. J. Hauke reports grants from AGO Research $\mathrm{GmbH}$ during the conduct of the study. A. du Bois reports personal fees from AstraZeneca, GSK/Tesaro, Roche, Genmab/Seattle Genetics, Zodiac, BIOCAD, and Clovis outside the submitted work. L.F. Wessels reports grants from Genmab BV outside the submitted work. E. Hahnen reports grants from AGO Research GmbH during the conduct of the study as well as other support from AstraZeneca outside the submitted work; in addition, E. Hahnen has a patent for BRCA1/2 classifiers pending. P. Harter reports grants from AstraZeneca during the conduct of the study as well as grants and personal fees from Immunogen and Clovis; personal fees from Stryker, Zai Lab, and MSD; and grants from Genmab, European Union, DKH, and DFG outside the submitted work. S.C. Linn reports grants from Starz Foundation and BBMRI-NL during the conduct of the study as well as grants and non-financial support from AstraZeneca, Genentech-Roche, Novartis, and Tesaro (now owned by GSK); grants from Eurocept-pharmaceuticals, Immunomedics; and other support from DaiichiSankyo and IBM outside the submitted work; in addition, S.C. Linn has a patent for BRCA-like ovarian cancer classifiers pending. R.K. Schmutzler reports grants from Ago Ovar study group during the conduct of the study as well as personal fees from AstraZeneca outside the submitted work. No disclosures were reported by the other authors.

\section{Authors' Contributions}

P.C. Schouten: Conceptualization, resources, data curation, software, formal analysis, validation, investigation, visualization, methodology, writingoriginal draft, project administration, writing-review and editing. L. Richters:

\section{References}

1. Fitzmaurice C, Abate D, Abbasi N, Abbastabar H, Abd-Allah F, Abdel-Rahman $\mathrm{O}$, et al. Global, regional, and national cancer incidence, mortality, years of life lost, years lived with disability, and disability-adjusted life-years for 29 Cancer Groups, 1990 to 2017: A systematic analysis for the global burden of disease study. JAMA Oncol 2019;5:1749-68.

2. National Cancer Institue. Cancer Stat Facts: ovarian cancer. Available from: $<$ https://seer.cancer.gov/statfacts/html/ovary.html $>$.

3. du Bois A, Reuss A, Pujade-Lauraine E, Harter P, Ray-Coquard I, Pfisterer J, et al. Role of surgical outcome as prognostic factor in advanced epithelial ovarian cancer: a combined exploratory analysis of 3 prospectively randomized phase 3 multicenter trials: by the Arbeitsgemeinschaft Gynaekologische Onkologie Studiengruppe Ovarialkarzinom (AGO-OVAR) and the Groupe d'Investiga-
Conceptualization, resources, data curation, formal analysis, validation, investigation, visualization, methodology, writing-original draft, project administration, writing-review and editing. D.J. Vis: Software, formal analysis, supervision, writing-review and editing. S. Kommoss: Resources, supervision, validation, project administration, writing-review and editing. E. van Dijk: Software, formal analysis, methodology, writing-review and editing. C. Ernst: Software, formal analysis, methodology, writing-review and editing. R.J.C. Kluin: Conceptualization, resources, data curation, software, formal analysis, methodology, writing-review and editing. F. Marmé: Resources, supervision, validation, project administration, writing-review and editing. E.H. Lips: Resources, formal analysis, supervision, project administration, writing-review and editing. S. Schmidt: Resources, data curation, formal analysis, validation, project administration, writing-review and editing. E. Scheerman: Resources, data curation, formal analysis, writing-review and editing. K. Prieske: Resources, supervision, validation, project administration, writing-review and editing. C.H.M. van Deurzen: Resources, data curation, formal analysis, supervision, writing-review and editing. A. Burges: Resources, supervision, validation, project administration, writing-review and editing. P.C. Ewing-Graham: Data curation, formal analysis, supervision, project administration, writing-review and editing. D. Dietrich: Resources, supervision, validation, project administration, writing-review and editing. A. Jager: Resources, supervision, project administration, writing-review and editing. N. de Gregorio: Resources, supervision, validation, project administration, writing-review and editing. J. Hauke: Resources, data curation, software, formal analysis, validation, investigation, methodology, project administration, writing-review and editing. A. du Bois: Resources, supervision, validation, project administration, writingreview and editing. P.M. Nederlof: Conceptualization, resources, data curation, formal analysis, supervision, methodology, project administration, writing-review and editing. L.F. Wessels: Resources, software, formal analysis, supervision, methodology, project administration, writing-review and editing. E. Hahnen: Resources, data curation, formal analysis, supervision, funding acquisition, validation, investigation, methodology, writing-original draft, project administration, writing-review and editing. P. Harter: Resources, supervision, validation, project administration, writing-review and editing. S.C. Linn: Conceptualization, resources, supervision, funding acquisition, validation, investigation, methodology, writing-original draft, project administration, writingreview and editing. R.K. Schmutzler: Resources, supervision, funding acquisition, validation, writing-original draft, project administration, writing-review and editing.

\section{Acknowledgments}

We would like to thank em. prof. dr. P.M.J.J. Berns for support and constructive feedback in the initial phase of the project. Collection of samples and DNA isolation of the training set were supported by BBMRI complementation project CP2011-40. The training of the classifiers was supported by an unrestricted research grant generously provided by the Starz Foundation. The AGO-TR1 trial was funded by AstraZeneca Germany and the AGO Research GmbH. L. Richters was supported by the program for Temporary Substitutes for Clinicians (Gerok substitute positions) at the Faculty of Medicine, University of Cologne.

The costs of publication of this article were defrayed in part by the payment of page charges. This article must therefore be hereby marked advertisement in accordance with 18 U.S.C. Section 1734 solely to indicate this fact.

Received May 6, 2021; revised September 12, 2021; accepted September 23, 2021; published first September 30, 2021.

teurs Nationaux Pour les Etudes des Cancers de l'Ovaire (GINECO). Cancer 2009;115:1234-44.

4. Piver MS. Treatment of ovarian cancer at the crossroads: 50 years after singleagent melphalan chemotherapy. Oncology 2006;20:1156,58.

5. Ledermann JA, Raja FA, Fotopoulou C, Gonzalez-Martin A, Colombo N, Sessa $\mathrm{C}$, et al. Newly diagnosed and relapsed epithelial ovarian carcinoma: ESMO Clinical Practice Guidelines for diagnosis, treatment and follow-up. Ann Oncol 2013;24:vi24-32.

6. Ledermann JA, Drew Y, Kristeleit RS. Homologous recombination deficiency and ovarian cancer. Eur J Cancer 2016;60:49-58.

7. Moynahan ME, Chiu JW, Koller BH, Jasin M. Brcal controls homology-directed DNA repair. Mol Cell 1999;4:511-8. 
8. Moynahan ME, Pierce AJ, Jasin M. BRCA2 is required for homology-directed repair of chromosomal breaks. Mol Cell 2001;7:263-72.

9. Moore K, Colombo N, Scambia G, Kim BG, Oaknin A, Friedlander M, et al. Maintenance olaparib in patients with newly diagnosed advanced ovarian cancer. N Engl J Med 2018;379:2495-505.

10. Robson M, Im SA, Senkus E, Xu B, Domchek SM, Masuda N, et al. Olaparib for metastatic breast cancer in patients with a germline BRCA mutation. $\mathrm{N}$ Engl J Med 2017;377:523-33.

11. Turner N, Tutt A, Ashworth A. Hallmarks of 'BRCAness' in sporadic cancers. Nat Rev Cancer 2004;4:814-9.

12. Lord CJ, Ashworth A. BRCAness revisited. Nat Rev Cancer 2016;16:110-20.

13. Ledermann J, Harter P, Gourley C, Friedlander M, Vergote I, Rustin G, et al. Olaparib maintenance therapy in patients with platinum-sensitive relapsed serous ovarian cancer: a preplanned retrospective analysis of outcomes by BRCA status in a randomised phase 2 trial. Lancet Oncol 2014;15:852-61.

14. Mirza MR, Monk BJ, Herrstedt J, Oza AM, Mahner S, Redondo A, et al. Niraparib maintenance therapy in platinum-sensitive, recurrent ovarian cancer. $\mathrm{N}$ Engl J Med 2016;375:2154-64.

15. Swisher EM, Lin KK, Oza AM, Scott CL, Giordano H, Sun J, et al. Rucaparib in relapsed, platinum-sensitive high-grade ovarian carcinoma (ARIEL2 Part 1): an international, multicentre, open-label, phase 2 trial. Lancet Oncol 2017;18: 75-87.

16. Coleman RL, Oza AM, Lorusso D, Aghajanian C, Oaknin A, Dean A, et al. Rucaparib maintenance treatment for recurrent ovarian carcinoma after response to platinum therapy (ARIEL3): a randomised, double-blind, placebo-controlled, phase 3 trial. Lancet 2017;390:1949-61.

17. Abkevich V, Timms KM, Hennessy BT, Potter J, Carey MS, Meyer LA, et al Patterns of genomic loss of heterozygosity predict homologous recombination repair defects in epithelial ovarian cancer. Br J Cancer 2012;107:1776-82.

18. Davies H, Glodzik D, Morganella S, Yates LR, Staaf J, Zou X, et al. HRDetect is a predictor of BRCA1 and BRCA2 deficiency based on mutational signatures. Nat Med 2017;23:517-25.

19. Birkbak NJ, Wang ZC, Kim JY, Eklund AC, Li Q, Tian R, et al. Telomeric allelic imbalance indicates defective DNA repair and sensitivity to DNA-damaging agents. Cancer Discov 2012;2:366-75.

20. Popova T, Manie E, Rieunier G, Caux-Moncoutier V, Tirapo C, Dubois T, et al. Ploidy and large-scale genomic instability consistently identify basal-like breast carcinomas with BRCA1/2 inactivation. Cancer Res 2012;72:5454-62.

21. Konstantinopoulos PA, Spentzos D, Karlan BY, Taniguchi T, Fountzilas E, Francoeur N, et al. Gene expression profile of BRCAness that correlates with responsiveness to chemotherapy and with outcome in patients with epithelial ovarian cancer. J Clin Oncol 2010;28:3555-61.

22. Timms KM, Abkevich V, Hughes E, Neff C, Reid J, Morris B, et al. Association of BRCA1/2 defects with genomic scores predictive of DNA damage repair deficiency among breast cancer subtypes. Breast Cancer Res 2014;16:475.

23. Moore KN, Secord AA, Geller MA, Miller DS, Cloven N, Fleming GF, et al Niraparib monotherapy for late-line treatment of ovarian cancer (QUADRA): a multicentre, open-label, single-arm, phase 2 trial. Lancet Oncol 2019;20:636-48.

24. Coleman RL, Fleming GF, Brady MF, Swisher EM, Steffensen KD, Friedlander $\mathrm{M}$, et al. Veliparib with first-line chemotherapy and as maintenance therapy in ovarian cancer. N Engl J Med 2019;381:2403-15.

25. Gonzalez-Martin A, Pothuri B, Vergote I, DePont Christensen R, Graybill W, Mirza MR, et al. Niraparib in patients with newly diagnosed advanced ovarian cancer. N Engl J Med 2019;381:2391-402.

26. Ray-Coquard I, Pautier P, Pignata S, Perol D, Gonzalez-Martin A, Berger R, et al. Olaparib plus bevacizumab as first-line maintenance in ovarian cancer. N Engl J Med 2019;381:2416-28.

27. European Medicines Agency. Lynparza, Opinion; 2020. Available from: $<\mathrm{https} / /$ www.ema.europa.eu/en/medicines/human/summaries-opinion/lynparza- $0>$.

28. Joosse SA, van Beers EH, Tielen IH, Horlings H, Peterse JL, Hoogerbrugge N, et al. Prediction of BRCA1-association in hereditary non-BRCA1/2 breast carcinomas with array-CGH. Breast Cancer Res Treat 2009;116:479-89.

29. Tibshirani R, Hastie T, Narasimhan B, Chu G. Diagnosis of multiple cancer types by shrunken centroids of gene expression. Proc Natl Acad Sci U S A 2002;99: $6567-72$.

30. Vollebergh MA, Lips EH, Nederlof PM, Wessels LF, Schmidt MK, van Beers EH, et al. An aCGH classifier derived from BRCA1-mutated breast cancer and benefit of high-dose platinum-based chemotherapy in HER2-negative breast cancer patients. Ann Oncol 2011;22:1561-70.

31. Vollebergh MA, Lips EH, Nederlof PM, Wessels LF, Wesseling J, Vd Vijver MJ, et al. Genomic patterns resembling BRCA1-and BRCA2-mutated breast cancers predict benefit of intensified carboplatin-based chemotherapy. Breast Cancer Res 2014;16:R47.

32. Schouten PC, Marme F, Aulmann S, Sinn HP, van Essen HF, Ylstra B, et al. Breast cancers with a BRCA1-like DNA copy number profile recur less often than expected after high-dose alkylating chemotherapy. Clin Cancer Res 2015;21 763-70.

33. Schouten PC, Gluz O, Harbeck N, Mohrmann S, Diallo-Danebrock R, Pelz E et al. BRCA1-like profile predicts benefit of tandem high dose epirubicincyclophospamide-thiotepa in high risk breast cancer patients randomized in the WSG-AM01 trial. Int J Cancer 2016;139:882-9.

34. Vliek S, Jager A, Jonge-Lavrencic M, Lotz J-P, Gonçalves A, Graeser M, et al. Abstract OT2-07-08: Substantially improving the cure rate of high-risk BRCA1like breast cancer patients with personalized therapy (SUBITO) - an international randomized phase III trial. Cancer Res 2018;78:OT2-07-08.

35. Severson TM, Wolf DM, Yau C, Peeters J, Wehkam D, Schouten PC, et al. The BRCA1ness signature is associated significantly with response to PARP inhibitor treatment versus control in the I-SPY 2 randomized neoadjuvant setting. Breast Cancer Res 2017;19:99.

36. Harter P, Hauke J, Heitz F, Reuss A, Kommoss S, Marme F, et al. Prevalence of deleterious germline variants in risk genes including BRCA1/2 in consecutive ovarian cancer patients (AGO-TR-1). PLoS One 2017;12:e0186043.

37. Hauke J, Hahnen E, Schneider S, Reuss A, Richters L, Kommoss S, et al. Deleterious somatic variants in 473 consecutive individuals with ovarian cancer results of the observational AGO-TR1 study (NCT02222883). J Med Genet 2019; 56:574-80.

38. Federation of Dutch Medical Scientific Societies. Human tissue and medical research: code of conduct for responsible use (2011); 2011. Available from $<$ https://www.federa.org/sites/default/files/images/print_version_code_of_con duct_english.pdf>.

39. Schouten PC, Grigoriadis A, Kuilman T, Mirza H, Watkins JA, Cooke SA, et al. Robust BRCA1-like classification of copy number profiles of samples repeated across different datasets and platforms. Mol Oncol 2015;9:1274-86.

40. Schouten PC, van Dyk E, Braaf LM, Mulder L, Lips EH, de Ronde JJ, et al. Platform comparisons for identification of breast cancers with a BRCA-like copy number profile. Breast Cancer Res Treat 2013;139:317-27.

41. Hastie T, Tibshirani R, Friedman J. The elements of statistical learning. New York, NY: Springer; 2009.

42. Bell D, Berchuck A, Birrer M, Chien J, Cramer DW, Dao F, et al. Integrated genomic analyses of ovarian carcinoma. Nature 2011;474:609-15.

43. Domanska K, Malander S, Staaf J, Karlsson A, Borg A, Jonsson G, et al. Genetic profiles distinguish different types of hereditary ovarian cancer. Oncol Rep 2010; 24:885-95.

44. Kamieniak MM, Munoz-Repeto I, Rico D, Osorio A, Urioste M, Garcia-Donas J, et al. DNA copy number profiling reveals extensive genomic loss in hereditary BRCA1 and BRCA2 ovarian carcinomas. Br J Cancer 2013;108:1732-42.

45. Maxwell KN, Wubbenhorst B, Wenz BM, De Sloover D, Pluta J, Emery L, et al BRCA locus-specific loss of heterozygosity in germline BRCA1 and BRCA2 carriers. Nat Commun 2017;8:319.

46. Macintyre G, Goranova TE, De Silva D, Ennis D, Piskorz AM, Eldridge M, et al. Copy number signatures and mutational processes in ovarian carcinoma. Nat Genet 2018;50:1262-70.

47. Prahallad A, Sun C, Huang S, Di Nicolantonio F, Salazar R, Zecchin D, et al. Unresponsiveness of colon cancer to BRAF(V600E) inhibition through feedback activation of EGFR. Nature 2012;483:100-3.

48. Alexandrov LB, Kim J, Haradhvala NJ, Huang MN, Tian Ng AW, Wu Y, et al. The repertoire of mutational signatures in human cancer. Nature 2020;578:94-101.

49. Watkins JA, Irshad S, Grigoriadis A, Tutt AN. Genomic scars as biomarkers of homologous recombination deficiency and drug response in breast and ovarian cancers. Breast Cancer Res 2014;16:211.

50. Castroviejo-Bermejo M, Cruz C, Llop-Guevara A, Gutiérrez-Enríquez S, Ducy $\mathrm{M}$, Ibrahim YH, et al. A RAD51 assay feasible in routine tumor samples calls PARP inhibitor response beyond BRCA mutation. EMBO Mol Med 2018;10: e9172. 


\section{Clinical Cancer Research}

\section{Ovarian Cancer-Specific BRCA-like Copy-Number Aberration Classifiers Detect Mutations Associated with Homologous Recombination Deficiency in the AGO-TR1 Trial}

Philip C. Schouten, Lisa Richters, Daniel J. Vis, et al.

Clin Cancer Res 2021;27:6559-6569. Published OnlineFirst September 30, 2021.

\section{Updated version Access the most recent version of this article at: doi:10.1158/1078-0432.CCR-21-1673}

Supplementary Access the most recent supplemental material at:

Material http://clincancerres.aacrjournals.org/content/suppl/2021/09/28/1078-0432.CCR-21-1673.DC1

Cited articles This article cites 46 articles, 7 of which you can access for free at: http://clincancerres.aacrjournals.org/content/27/23/6559.full\#ref-list-1

E-mail alerts Sign up to receive free email-alerts related to this article or journal.

Reprints and To order reprints of this article or to subscribe to the journal, contact the AACR Publications Department at Subscriptions pubs@aacr.org.

Permissions To request permission to re-use all or part of this article, use this link http://clincancerres.aacrjournals.org/content/27/23/6559.

Click on "Request Permissions" which will take you to the Copyright Clearance Center's (CCC) Rightslink site. 